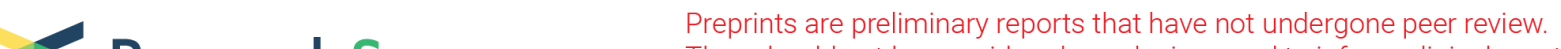 $\begin{array}{ll}\text { Research Square } & \text { They should not be considered conclusive, used to inform clinical practice, } \\ \text { or referenced by the media as validated information. }\end{array}$
}

\section{An Efficient Parallel Block Compressive Sensing Scheme for Medical Signals and Image Compression}

Parnasree Chakraborty ( $\sim$ prernasree@bsauniv.ac.in )

BSA CRESCENT INSTITUTE OF SCIENCE \& TECHNOLOGY https://orcid.org/0000-0003-1093-0685

Tharini $C$

BSA Crescent Institute of Science and Technology

\section{Research Article}

Keywords: Compressed Sensing, Parallel Processing, Data compression, Peak signal to noise ratio, Image reconstruction, Image quality

Posted Date: June 29th, 2021

DOI: https://doi.org/10.21203/rs.3.rs-378554/v1

License: (c) (i) This work is licensed under a Creative Commons Attribution 4.0 International License.

Read Full License

Version of Record: A version of this preprint was published at Wireless Personal Communications on October 28th, 2021. See the published version at https://doi.org/10.1007/s11277-021-09270-w. 


\title{
An Efficient Parallel Block Compressive Sensing Scheme for Medical Signals and Image Compression
}

\author{
Parnasree Chakraborty ${ }^{1}$ and Tharini $\mathrm{C}^{2}$ \\ ${ }^{1,2}$ BSA Crescent Institute of Science \& Technology, Chennai, India \\ Email: prernasree@crescent.education \\ Orcid id: https://orcid.org/0000-0003-1093-0685
}

\begin{abstract}
With rapid development of real-time and dynamic application, Compressive Sensing or Compressed sensing (CS) has been used for medical image and biomedical signal compression in the last decades. The performance of CS based compression is mostly dependent on decoding methods rather than the CS encoding methods used in practice. Many CS encoding and decoding algorithms have been reported in literature. However, the comparative study on performance metrics of CS encoding with block processing and without block processing is not investigated by the researchers so far. This paper proposes block CS based medical images and signals compression technique and the proposed technique is compared with standard CS compression. The proposed algorithm divides the input medical images and signals to blocks and each block is processed parallel to enable faster computation. Three performance indices, i.e., the peak signal to noise ratio (PSNR), reconstruction time (RT) and structural similarity index (SSIM) were tested to observe their changes with respect to compression ratio. The results showed that block CS algorithm had better performance than standard CS based compression. More specifically, the parallel block CS reported the best results than standard CS with respect to less reconstruction time and satisfactory PSNR and SSIM.
\end{abstract}

Index Terms - Compressed Sensing, Parallel Processing, Data compression, Peak signal to noise ratio, Image reconstruction, Image quality

\section{INTRODUCTION}

A signal processing technique known as Compressive Sensing or Compressed Sensing (CS) is used in almost all the field of communication engineering in recent years. For efficient acquisition and reconstruction of a signal, CS can be used as a data reduction tool. In CS based reconstruction, underdetermined linear systems [1] are solved using linear programming and by finding solutions to the underdetermined system, perfect recovery of the signal is done. In [2], it is explained very well how the CS theory can be used to recover signals from very few number of samples (measurements) than traditional and conventional methods. The working principle of CS is completely different than the normal sampling theorem. The normal sampling theorem says that for successful reconstruction of any signal, the sampling rate must be atleast twice the number of highest frequency component present in the signal [3].

According to CS theory, the conventional sampling theorem need not be followed. To make this possible, CS relies on two principles: one is Sparsity and the other principle is incoherence. Sparsity [1], depends on the density of the signal and incoherence, which depends on the sensing modality. Both the principles are discussed in detail as follows.

\section{A. Sparsity}

A sparse matrix is a matrix or a sparse array is an array in which few elements are non-zero and most of the elements are zero. On the other hand, most of the elements are nonzero in a dense matrix. The parameter sparsity can be found by finding the number of zerovalue elements divided by the total number of elements in a matrix or array. The sparsity can also be found by subtracting the density of the matrix from unity. In general many signals are sparse or compressible by nature. This behaviour of signal is exploited by CS theory. The meaning is, the signals have sparser representations when they are expressed in the proper basis ' $\Psi^{\prime}$. Mathematically if we have a vector ' $\mathrm{x}$ ' of length ' $\mathrm{N}$ ' (i.e. $\mathrm{x} \in \mathrm{R}^{\mathrm{N}}$ ), then the vector ' $\mathrm{x}$ ' can be expanded using an orthonormal basis $\Psi=\left[\Psi_{1} \Psi_{2} \ldots . . . \Psi \mathrm{N}\right]$ using the equation (1).

$$
\mathrm{s}=\sum_{i=1}^{N} \mathrm{xi} \Psi \mathbf{i}
$$

Where sparse representation of input signal ' $\mathrm{x}$ ' is denoted by ' $\mathrm{s}$ '. The number of non-zero components in the vector 's' are much lesser than the non-zero components in vector ' $x$ ' if the spreading basis is sparse. From equation (1) it is clear that when a signal has a sparse representation, small coefficients can be discarded without much perceptual loss.

\section{B. Incoherence}

Incoherence principle is extended by the duality between time and frequency. It expresses the idea that objects having a sparse 
representation in a basis ' $\Psi$ ' must be spreaded out in the domain in which they are acquired. The concept of incoherence can be made much clear with the help of a spike signal or impulse signal. In the time domain a Dirac or a spike signal is concentrated to one particular time instant whereas when the same signal is represented in frequency domain, it spreads out in the frequency domain. Similar concept can be used in CS based sparse representation of the signal. Suppose given a pair of $(\Phi, \Psi)$ orthobases of dimension $\mathrm{R}^{\mathrm{n}}$ each. For explanation of incoherence let ' $\Phi$ ' basis is used for sensing the signal ' $\mathrm{x}$ ' and the other basis is used to represent ' $\mathrm{x}$ ' in sparse domain. The relation between these two bases decides the reconstruction quality in CS recovery and hence it is essential to determine the degree of correlation between the pair of orthobases. The mutual coherence of a pair of orthobases $(\Phi, \Psi)$ is the measure of highest similarity factor between any two columns of ' $\Psi^{\prime}$ and ' $\Phi$ '. If ' $\Phi$ ' and ' $\Psi$ ' contain correlated elements, the coherence is large, otherwise the mutual coherence value is small.

The mutual coherence between the sensing basis ' $\Phi$ ' and the representation basis ' $\Psi$ ' is given by the equation (2).

$$
\mu(\Phi, \Psi)=\sqrt{\mathrm{n}} \cdot \max (\Phi \mathrm{k}, \Psi \mathrm{j})
$$

Where, $1 \leq \mathrm{k}, \mathrm{j} \leq \mathrm{n}$;

In CS based reconstruction, small mutual coherence value is desirable as it provides better recovery of the signal.

\section{CS ENCODING}

The CS algorithm can be better explained with the help of mathematical expressions given in this section. Conversion of the acquired medical signals or images into a sparse signal is done by the use of sparsifying kernel in CS. Sparse signals are the signals which are very few in number and has complete structure and meaningful information which are essential to reconstruct the original signal or image. The generation of the sparse signal (s) in matrix form is given in equation (3).

$$
\mathrm{s}=\Psi \mathrm{X}
$$

In equation 3, the input signal is denoted by ' $\mathrm{x}$ ' and' $\mathrm{s}$ ' is the sparse representation of the input data and ' $\Psi$ ' is the transform matrix or kernel. After sparse generation, measurement matrix ' $A$ ' is used for compression of the sparse signal or image. The output ' $y$ ' which is the compressed version of input ' $x$ ' is given in equation (4),

$$
\mathrm{y}=\mathrm{As}
$$

Where ' $A$ ' is called the measurement or sensing matrix, ' $\mathrm{s}$ ' is the sparse representation and ' $\mathrm{y}$ ' is the compressed output.

\section{CS RECONSTRUCTION}

CS involves a variety of methods for representation of a signal on the basis of a limited number of samples. CS based recovery of signals involve reconstruction of signal from these reduced number of samples. The major issue in CS recovery [4] is how to effectively reconstruct the original signal from the compressed data without the loss of generality. Currently, there are varieties of reconstruction algorithms available in literatures which are defined either based on the framework of convex optimization, or greedy approaches. These are namely: Convex Relaxation, Non Convex Minimization Algorithms, Greedy Iterative Algorithm, Combinatorial / Sublinear Algorithms, Iterative Thresholding Algorithms, Bregman Iterative Algorithms.

The complexity of the CS recovery algorithm and also the computational speed of the algorithms are very important as these factors influence the recovery of the signal in practical situations. In general, the convex relaxation method is used for CS reconstruction for its simplicity and computational speed. Convex relaxation [5] based recovery method has high computational speed compared to other reconstruction algorithms mentioned above. The convex relaxation reconstruction method is used in this work.

Parallel CS based medical image compression is suggested in this paper. In order to compress very large dimensional image, block CS is much suitable technique in which the image is splitted into number of blocks and each block is compressed simultaneously to perform compression. The reconstruction result obtained is very good in quality and hence block CS is suitable for medical image compression, especially in real time application. An effective algorithm to compress and to reconstruct digital images is presented in this paper. The comparison of compression methods block CS and standard CS on the basis of compression ratio and compression quality is the outline of this work. 
In order to compress very large dimension medical image and long duration brain signal, block CS is suggested in which the input signal is divided into number of blocks and each block is compressed simultaneously to perform compression. Medical images of size $(\mathrm{M} \times \mathrm{N})$ each is considered and taken from database [6]. The size of row ' $\mathrm{M}$ ' and size of column ' $\mathrm{N}$ ' are divided by 4 and blocks are generated. Preferably the row and column size ' $\mathrm{M}$ ' and ' $\mathrm{N}$ ' must be of multiple of 4 . If not than zero padding is done to make row and column size of image that to be compressed are of multiple of 4. Each block is separately compressed using standard compressed sensing algorithm [7]. The reconstruction result shows very good quality. The steps involved in block CS for a signal of size (M x N) is shown in figure. At the first step, an original data of size ( $\mathrm{M} \times \mathrm{N})$ is considered then zero Padding is done to make the size $\mathrm{M}$ ' and ' $N$ ' multiple of 4 if they are not multiple of 4 and divided into ' $n$ ' number blocks of each size (M/4 X N/4). Parallel Processing of each blocks for CS based compression is performed and reconstruction of each block is performed. At last step all the reconstructed blocks are combined to get final reconstructed signal and image.

\section{RESULTS AND DISCUSSIONS}

Figure 1(a), 2(a) and 3(a) shows the input sample medical image and brain signal of size ( $\mathrm{M} x \mathrm{~N})$ matrix. This image /signal is then compressed by both standard CS and block CS and the compression ratio is maintained 2:1. From the compressed pixel values, the data is reconstructed [8] and shown in figure below.

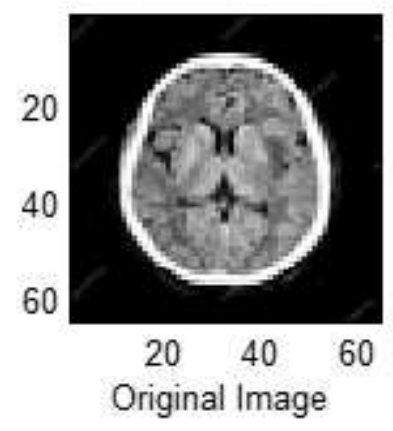

Figure 1(a). Sample Image of size (64x64)

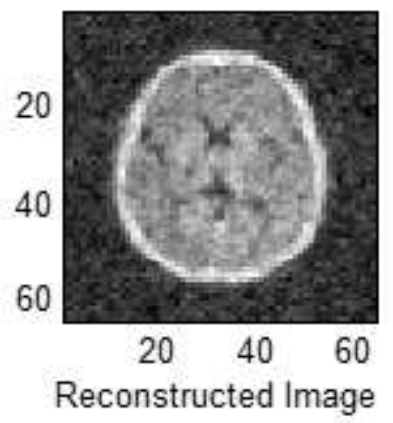

Figure 1(b). Reconstructed Image of size (64x64) using standard CS

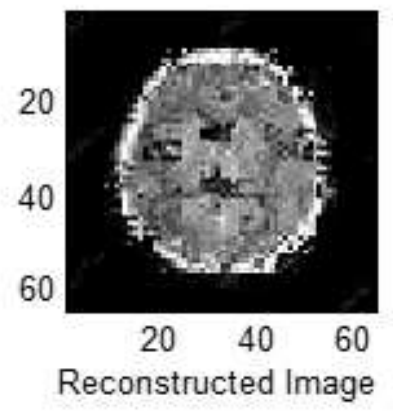

Figure 1(c). Reconstructed Image of size (64x64) using Parallel processing 


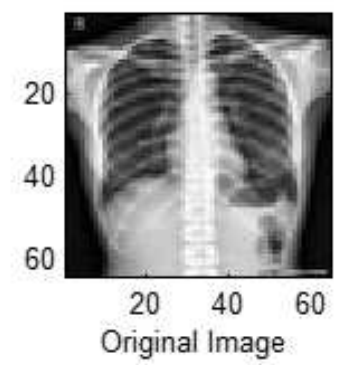

Figure 2(a). Sample Lung X-ray image of size(64 x 64)

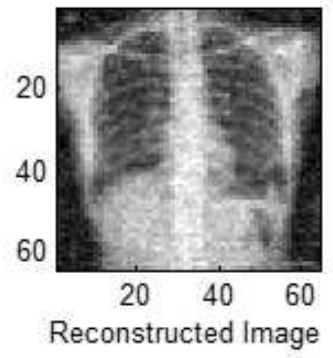

Figure 2(b). Reconstructed image of size (64 x 64) using standard CS

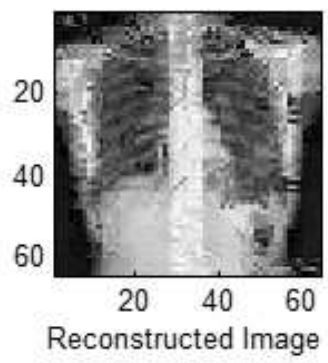

Figure 2(c). Reconstructed image of size (64 x 64) using parallel processing CS

The brain signal is considered as next input to the both algorithms and the original \& reconstructed signals are shown in following Figures 3(a), 3(b) and 3(c).

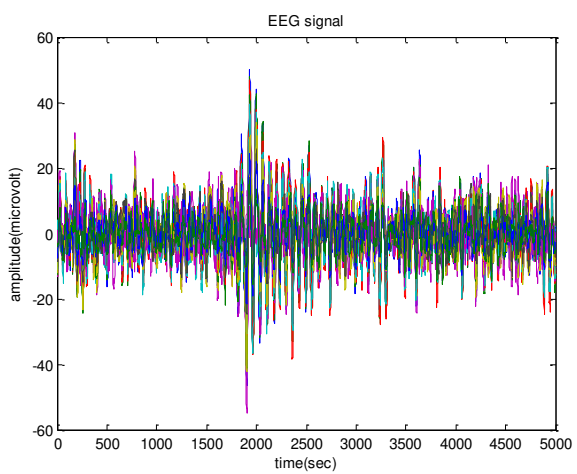

Figure 3(a). Sample brain signal of size (5000 x 16) 


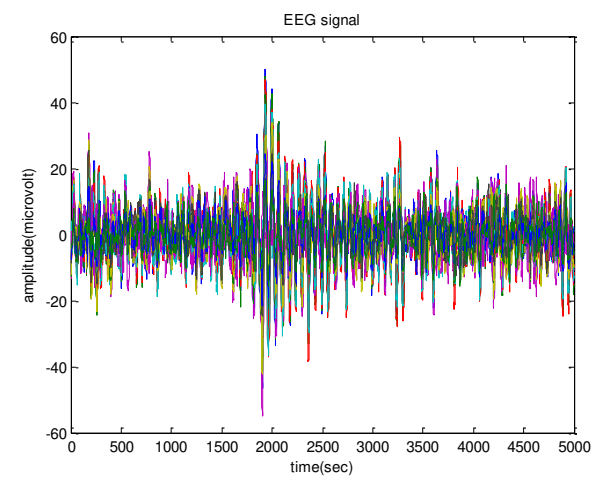

Figure 3(b). Reconstructed brain signal of size (5000 x 16) using standard CS

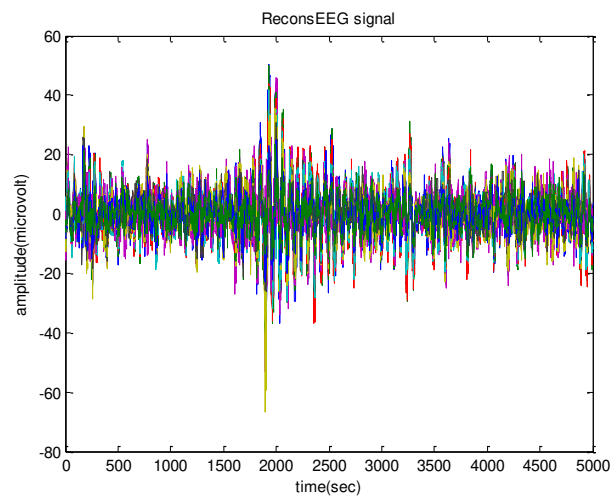

Figure 3(c). Reconstructed brain signal of size (5000 x 16) using parallel processing CS

The images / signal reconstructed with 50\% pixels using block compressed sensing is shown in Figure 2. The quality of both the original and reconstructed image can be best measured by calculating the Peak Signal-to-Noise Ratio (PSNR), reconstruction time (RT) and structural similarity index (SSIM) which are shown in tables.

PSNR values of input and reconstructed image/ signal indicate that both the images / signal are of equal quality and hence CS can be used for compression of large medical images $[9,11]$.

TABLE1. PERFORMANCE INDICES OF STANDARD CS AND BLOCK CS ON SAMPLE BRAIN CT SCAN IMAGE

\begin{tabular}{ccccc}
\hline \hline $\begin{array}{c}\text { Types of } \\
\text { Compression } \\
\text { Technique }\end{array}$ & $\begin{array}{c}\text { Sampling } \\
\text { Ratio }\end{array}$ & $\begin{array}{c}\text { PSNR } \\
\text { in dB }\end{array}$ & $\begin{array}{c}\text { RT in } \\
\text { Seconds }\end{array}$ & SSIM \\
\hline Standard CS & $2: 1$ & 31.348 & 124.150 & 0.6188 \\
Block CS & $2: 1$ & 31.092 & 1.0285 & 0.6916 \\
\hline \hline
\end{tabular}

The above table shows the measured PSNR values in $\mathrm{dB}$. 
TABLE2. PERFORMANCE INDICES OF STANDARD CS AND BLOCK CS ON SAMPLE LUNG X-RAY IMAGE

\begin{tabular}{ccccc}
\hline \hline $\begin{array}{c}\text { Types of } \\
\text { Compression } \\
\text { Technique }\end{array}$ & $\begin{array}{c}\text { Sampling } \\
\text { Ratio }\end{array}$ & $\begin{array}{c}\text { PSNR } \\
\text { in dB }\end{array}$ & $\begin{array}{c}\text { RT in } \\
\text { Seconds }\end{array}$ & SSIM \\
\hline Standard CS & $2: 1$ & 30.012 & 124.752 & 0.795 \\
Block CS & $2: 1$ & 29.285 & 1.134 & 0.717 \\
\hline \hline
\end{tabular}

TABLE3. PERFORMANCE INDICES OF STANDARD CS AND BLOCK CS ON SAMPLE BRAIN SIGNAL

\begin{tabular}{ccccc}
\hline \hline $\begin{array}{c}\text { Types of } \\
\text { Compression } \\
\text { Technique }\end{array}$ & $\begin{array}{c}\text { Sampling } \\
\text { Ratio }\end{array}$ & $\begin{array}{c}\text { PSNR } \\
\text { in dB }\end{array}$ & $\begin{array}{c}\text { RT in } \\
\text { Seconds }\end{array}$ & SSIM \\
\hline Standard CS & $2: 1$ & 35.968 & 62.399 & 0.151 \\
Block CS & $2: 1$ & 37.224 & 15.785 & 0.633 \\
& & & & \\
\hline \hline
\end{tabular}

\section{CONCLUSION}

Results of this work shows that even for $50 \%$ compression ratio, the performance of block CS based compression is superior than its counterpart standard CS. Block compressed sensing based compression algorithm is compared with standard CS based compression with respect to the reconstruction quality. Various performance measuring parameters are computed for proposed compression method. Especially compression of large image using block CS provides best results compared to standard method of CS based compression and hence block CS can be used for compression of images in real time application because of the high speed of the suggested algorithm.

\section{CONFLICT OF INTEREST}

The authors declare no conflict of interest.

\section{AUTHOR CONTRIBUTIONS}

Parnasree Chakraborty conducted the research and analyzed the data; Tharini. C wrote the paper. All authors had approved the final version.

\section{ACKNOWLEDGMENT}

The authors wish to thank BSACIST for providing the proprietary software to carry out the simulation and test the results in the research laboratory. 


\section{REFERENCES}

[1] Evaggelia Tsiligianni, Lisimachos P. Kondi , Aggelos K. Katsaggelos, ” Preconditioning for Underdetermined Linear Systems with Sparse Solutions", IEEE Signal Processing Letters, Vol.22, 2015, pp.1239- 1243.

[2] Emmanuel J. Candes, Michael B. Wakin,"An Introduction to Compressive Sampling”, IEEE Signal Processing Magazine, Vol. 25, 2010 , pp. 21 - 30.

[3] Luke, H.D,”The origins of the sampling theorem”, IEEE Communications Magazine, Vol. 37(4), 1999, pp. 106-108.

[4] Needell, D., Tropp, J.A.," CoSaMP: Iterative signal recovery from incomplete and inaccurate samples", Applied and Computational Harmonic Analysis, Vol. 26(3), 2009, pp.301-321.

[5] Scott Shaobing Chen, David L. Donoho, Michael A. Saunders,” Atomic Decomposition by Basis Pursuit”SIAM REVIEW, Vol. 43, 2001 , pp. 129-159.

[6] "Standard Test Images". [Online]. Available:https://www.ece.rice.edu/ wakin/images/

[7] David L. Donoho, ”Compressed sensing", IEEE Transactions on Information Theory, Vol.52, 2009, pp. 1289 - 1306.

[8] Liu Yan, H Zhou, H Zhang, T Liu, "Image compression using compressed sensing based on empirical mode decomposition", The Journal of China Universities of Posts and Telecommunications, Elsevier Publications, Vol.18,2011,pp. 123-128.

[9] Smitha Joyce Pinto, Jayanand, P. Gawande,” Performance analysis of medical image compression techniques“, 2012, 2012 Third Asian Himalayas International Conference on Internet, Kathmandu, Nepal.

[10] Parnasree Chakraborty, C. Tharini, “Analysis of Suitable Modulation Scheme for Compressive Sensing Algorithm in Wireless Sensor Network”, Sensor Review (ISSN: 0260-2288), Emerald Group Publishing Limited, Vol. 35, Issue. 2, 2015, pp.168-173.

[11] Parnasree Chakraborty ,Tharini Prasad, "An Efficient Communication Protocol For Wireless Sensor Network Using Differential Encoding Based Compressed Sensing Technique", International Journal Of Circuits, Systems and Signal Processing ( ISSN: 1998-4464), NAUN publication, Vol. 12,2018, pp.356-366. 